\title{
Orthodontic Photography: Know Your Camera
}

\author{
Pooja Kapoor ${ }^{1}$, Harpreet Singh ${ }^{2 *}$ and Jagvinder Singh Mann ${ }^{3}$ \\ ${ }^{1}$ Department of Orthodontics and Dentofacial Orthopaedics, MNDAV Dental college, India \\ ${ }^{2}$ Department of Conservative Dentistry and Endodontics, MNDAV Dental college , India \\ ${ }^{3}$ Department of Conservative Dentistry and Endodontics, Government Dental College and Hospital, India
}

*Corresponding author: Harpreet Singh, Professor and Head, Department of Conservative Dentistry and Endodontics, MNDAV Dental college , India, Tel: 98154893618; Email: hsgentledental@gmail.com

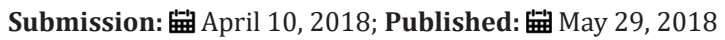

\begin{abstract}
Photography is a universal means of communication and a valuable tool in many fields to record the things we see, as well as many subjects beyond our range of vision. In skilful hands, a camera transforms an ordinary scene into an image of exceptional beauty. Obtaining high quality photographs is often tough even in the hands of the most enthusiastic photographers. This article focuses on making the orthodontists more aware of the sophisticated equipment called a camera and its accessories without which, our orthodontic records are unfeasible.
\end{abstract}

Keywords: Camera; Digital; Orthodontics; Photography

\section{Introduction}

Photography is a unique means of communication and a highly valuable tool in the field of medical and dental science, not only for record keeping but for education and motivation of patients as well. The word 'photograph' comes from Greek Work (phoslight, Graphein-to draw) meaning to write or draw with light [1]. Obtaining high quality photographs is often tough even in the hands of the most enthusiastic hospital photographers.

The complete process by which pictures are made by the chemical action of light on a sensitized plate or film is known as photography [2]. Many chemicals are sensitive to and affected by light (e.g. silver chloride salt darkens by prolonged exposure to light).

\section{History}

Photography was not discovered by any single person. As far back as $14^{\text {th }}$ century, ancient Greek Philosopher Aristotle happened to observe a true but inverted image of the outside scene on the opposite wall of a darkened room [3]. In 1569, Della Porta of Italy discovered the possibilities of using a glass lens for a sharp projection of the outside landscape [4].

In 1727, a German physicist Johann H Schulye discovered that silver salts turn dark when exposed to light. About 50 years later, Carl Scheeje, a Swedish chemist, showed that these changes in the salts by light could be made permanent by chemical treatment. In the late $18^{\text {th }}$ century, Sir John Herschel discovered sodium thyosulphate as a fixing agent. In 1824, Joseph Nicephore of France coated glass with a layer of bitumen dissolved in oil of lavender to obtain a negative resulting in the world's first photograph that showed the view from Joseph's window.

In 1839, Daguerre accidently discovered the first developing agent which he published on request of the French Govt. and named it Daguerreotype [5]. In 1841, WH Fox Talbot prepared a photography paper for making negatives and positives (process was called Talbotype or Calotype). In 1849, he prepared the glossy type paper. Scott Archer of London, 1851 introduced his collodion wet plate method of making negatives and Dr. RL Maddox, 1871 invented the present gelatin dry plate.

In 1898, Reverend Hannibal Goodwin of America introduced roll films commercially prepared by Eastman Kodak Company for the first time. Leica Company, 1924 introduced a 33mm camera small enough to fit in a pocket [6]. The elective flash bulb (1929) and electronic flash bulb (1931), greatly expanded the range of photographic subjects. Color film was first commercially produced in 1935.

\section{The Camera}

The term camera is shortened from camera obscura, literally "dark room" in Latin [7]. It is basically a box with an aperture, lens attached at one end and film at the other (Figure 1).

Principle: It works in much the same way as our eye. The eye lens focuses the image on to the nerve cells in retina which is sent to the brain by the optic nerve (Figure 2). The camera lens similarly 
focuses the light reflecting from subject into an image on the film. Light rays from the top of the subject form the lower part of the

image (upside down) [8] as represented in Figure 3. Most of the

cameras consist of four main parts, viz., body, lens, shutter and view-finder

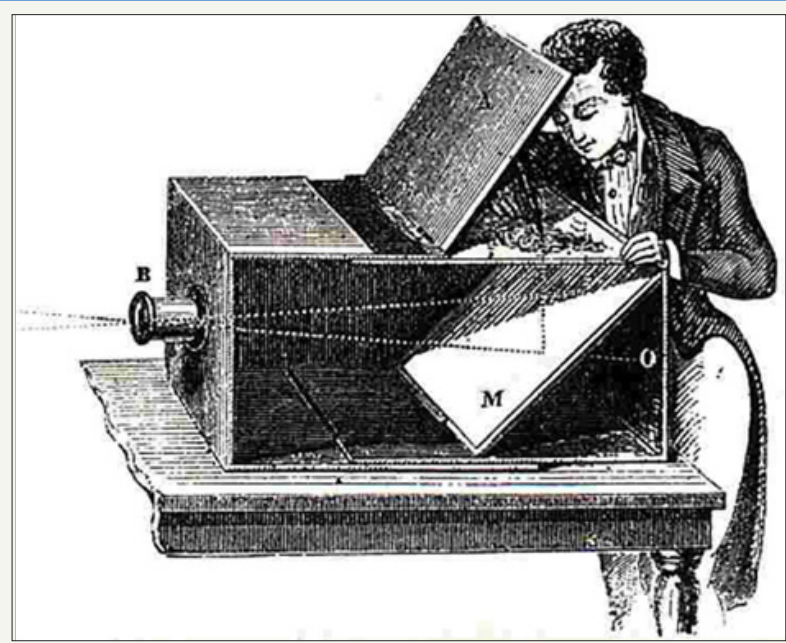

Figure 1: One of the first cameras.

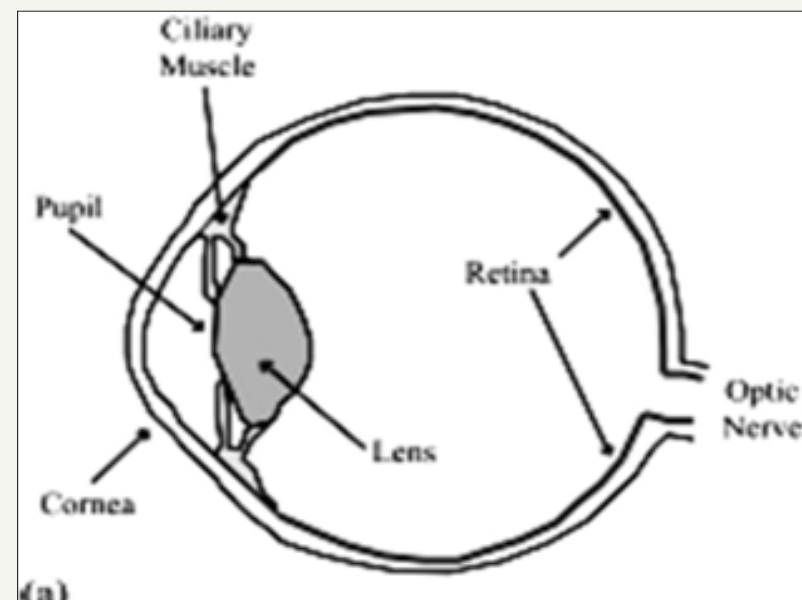

(a) (b)

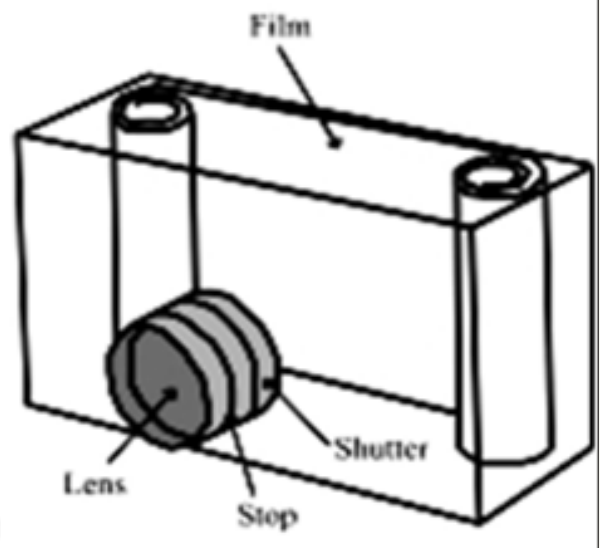

Figure 2: Eye is optically similar to a camera. (a) Human eye (b) camera.

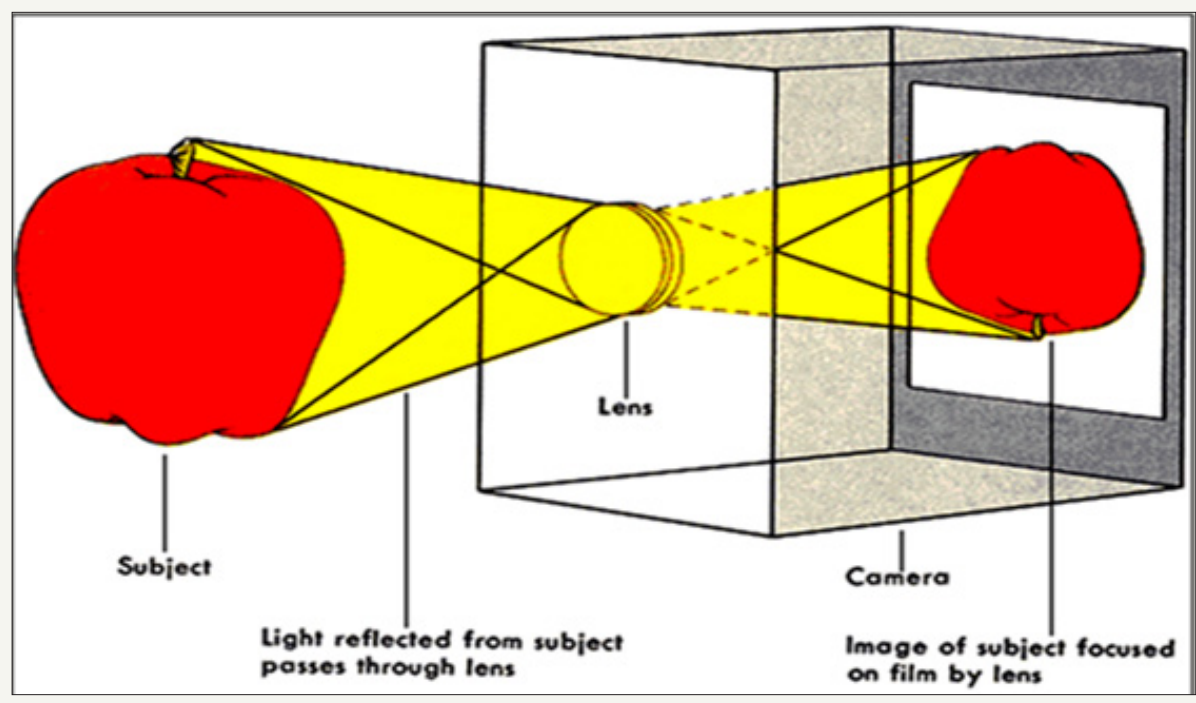

Figure 3: Light rays from the top of the subject form the lower part of the image (upside down). 
A. The body: Made of die-cast sheet metal or some synthetic material, it's light-tight, usually covered with leather or plastic for good finish.

B. The lens: (heart of the camera): It turns the three dimensional world outside the camera into a two dimensional image on the film inside.

Types of lenses [9]

i. Fisheye lens: These ultra wide angled lenses (focal length between $6-16 \mathrm{~m}$ ) have an extreme angle of view for shooting interiors or other confined spaces.

ii. Telephoto lens: With focal length greater than diagonal of the negative $(80 \mathrm{~mm}$ to $1,000 \mathrm{~mm}$ or even up to $2,000 \mathrm{~mm}$ ) and a very shallow depth of field, these are useful for pictorial, nature, candid and press photography.

iii. Wide angle lens: With focal length shorter than the diagonal of the negative $(17 \mathrm{~mm}$ to $35 \mathrm{~mm})$ and a much greater depth of field, they can create unusual effects. These cover more subject matter in smaller images like press photography.

iv. Zoom lens: These lenses (variable focal length) help to have quick change in the distance, without moving the camera. Telezooms are mostly available from $80 \mathrm{~mm}$ to $200 \mathrm{~mm}$ focal length.

v. Macro lens: These lenses work from infinity to a close-up of 3- 4 inches. 100-105mm macro lenses are generally used for dental photography. These are much sharper than zoom lenses because of fixed focal ability.

The shutter: It protects the film from light until the chosen moment. Hence, shutter speed is the length of the exposure time. It's a mechanical device, which passes the light through the lens of the camera so that an image is formed on the film. Mostly, shutters are of the following types: a) Rotating metal disc with a hole : in box and compact type cameras

b) Compound shutter, consisting of a number of overlapping steel blades, used in twin lens reflex, range-finder and large format cameras.

c) Focal plane shutter consisting of a cloth or metal blind with a moving slit, used in most of the single reflex cameras. These are of two types-mechanical (conventional with hand set shutter speed) and electronic (powered by an electromagnet with various electronically controlled shutter speeds).

The view-finder: It allows us to look at the subject through it and compose the picture area before shooting. Various types:

a) Waist level reflecting finder

b) Direct eye level finder

c) Ground glass and mirror view-finder

d) Prism, ground glass and mirror view-finder

Beside the above main parts of the camera, there are three main features of controlling the exposure and producing a sharp image.

Aperture or stop i.e. 'f': It's the opening through which controlled amount of light passes from the subject to strike the film. Aperture size is a measure of the size of opening measured as the ratio between focal length and the diameter of lens opening.

The aperture system serves as a universal measurement of the light- admitting capacity of any lens. It has a universal series which runs as: $\mathrm{f}-1.4,2,2.8,3.5,4 ; 5.6,8,11,16.22$ (Figure 4). The highest number viz. 22 in the series corresponds to the minimum aperture, allowing minimum light to pass through the lens and vice versa. The aperture is one of the most important factors for correct exposure [10]. Some lenses have a rotating ring on the lens barrel called the aperture selection ring. Other cameras have an electronic dial to control this setting.

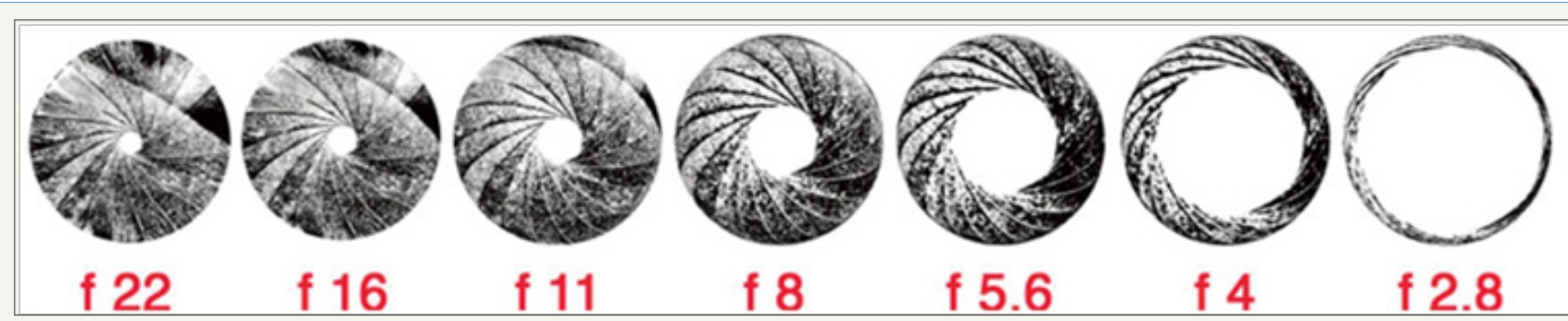

Figure 4: Aperture universal measurement system.

A. Shutter speed: The shutter has a two-fold function: to regulate the time during which light reaches the film (shutter speed); and to synchronize the flash contact. Shutter speeds run in a series and are marked in a number representing fractions of a second:

i) In older models of camera: $\mathrm{T}, \mathrm{B}, 1,2,5,10,25,50,100,250$, 500 . ii) In newer models: $\mathrm{B}, 1,2,4,8,15,30,60,125,250,500$, 1000, 2000, 4000, 8000 .

iii) In the box, instamatic and compact cameras, shutter speed is usually fixed at $1 / 125$ of a second.

Each speed will allow half as much of light to strike the film as the preceding one. For eg.1/30 will allow twice as much light 
as 1/60 would. Usually, in Dental Photography, we have standard static situations [11].

B. " $T$ " and "B" settings: $T$ represents very long time exposure while ' $\mathrm{B}$ ' represents brief or short exposure. When the shutter is set at ' $\mathrm{B}$ ' and pressed, it remains open till released. Nowadays, in cameras, only B is designed.

C. Focusing: Adjusting the correct distance between the lens and the object to get a sharp image on the film is called focusing which can be obtained by:

a) Intelligent guesswork (some old cameras);

b) Moving the lens forward or backward till image on the ground glass screen is sharp (reflex cameras); c) Fusing two split images into one till image becomes sharp (couple range-finder type cameras).

\section{Types of camera [12]}

Several types of cameras are available that are generally grouped as:

a) Simple box camera: These are roll film cameras fitted with a simple small lens, a fixed shutter speed and a fixed aperture of f.16 or f.11.

b) Simple miniature camera: These are generally of a fairly high standard, with a number of apertures and shutter speeds.

c) Compact aim and shoot $35 \mathrm{~mm}$ and 110 cameras: These latest non-interchangeable lens type cameras are good for snap shooting and record photography.

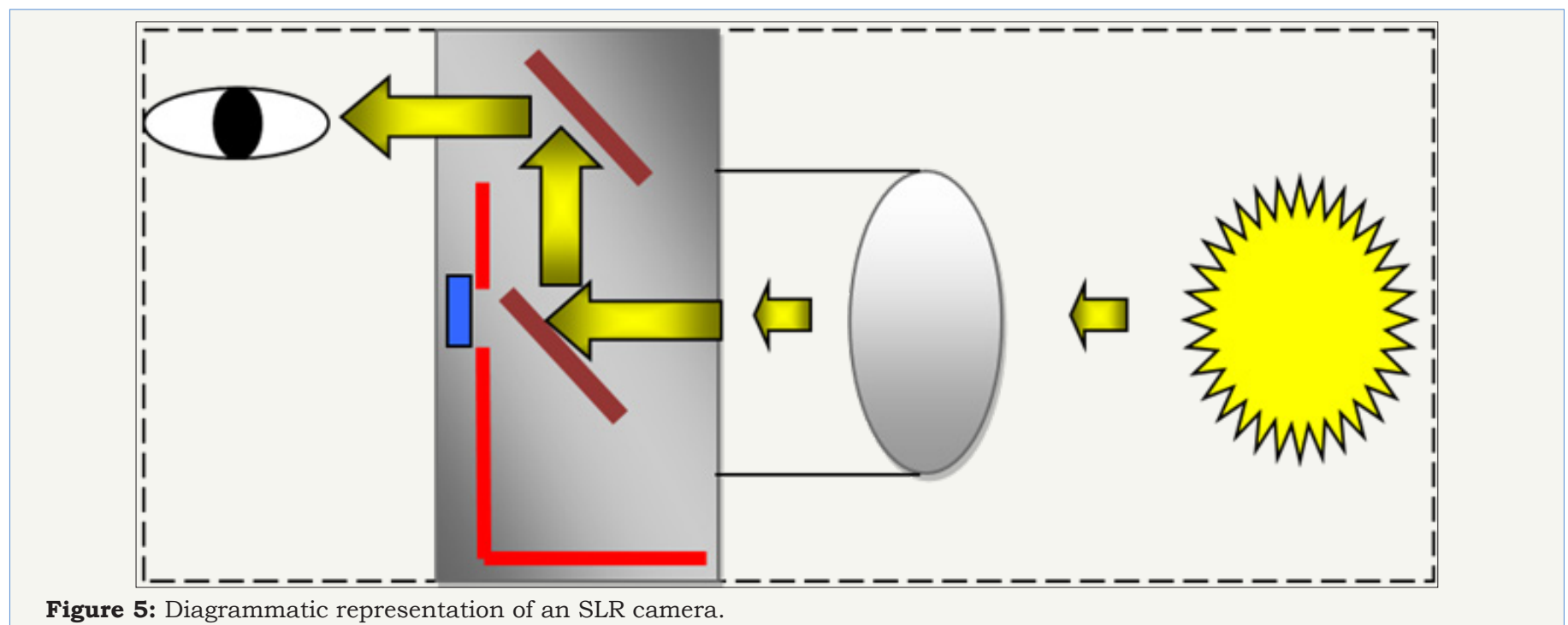

d) Modern reflex camera: Most popular cameras nowadays, these are compact, versatile and handy to use, main feature being its horizontal ground glass focusing screen on the top. There are two types of reflex camera single lens reflex and twin lens reflex (Figure 5).

Single Lens reflex (S.L.R. cameras): Most of them produce $24 \mathrm{~mm} \times 36 \mathrm{~mm}$ size negatives and some $6 \mathrm{~cm} \times 6 \mathrm{~cm}$. But they all have one common feature: What you see is exactly the picture you are making. Twin lens reflex cameras: two lenses of equal focal length are mounted rigidly together with their axis parallel and horizontal, upper lens for viewing and composing the picture and lower for making the actual image on film.

e) Sub-miniature and ultra-miniature camera: These are high-precision cameras having their own limitations. These are designed for $8 \mathrm{~mm}, 16 \mathrm{~mm}$ and some for $35 \mathrm{~mm}$ size films $(1 / 2$ frame) with automatic simultaneous shutter winding and film transport device.

f) Polaroid camera: Invented by Dr. Edwin Land of USA in 1947, these cameras produce instant prints within few seconds without the need of a dark room. Polaroid films are of two types: dry process film and peel apart type film, available in 3 $1 / 4$ " x 4 1/4" and $31 / 4 "$ x 33/8" sizes in either color or black and white.

It may be noted here that if one can afford and is really interested in the art of photography, one should get a good modern camera with all control devices, rather than first spend hard-earned money on a cheap, inferior camera.

\section{Depth of Field}

This is the zone of sharpness that extends in front of and behind the subject in which the image is sharp. The depth of field of any lens increases when:

a) Distance between the object and the lens increases.

b) Focal length of lens decreases and

c) Aperture is stepped down.

The depth of field does not extend equally in front of, and behind, the point of focus but approximately $1 / 3^{\text {rd }}$ in front of the point of focus, and $2 / 3^{\text {rd }}$ behind it [13]. It is maximum when the aperture is minimum and vice versa. 


\section{Resolving power}

It is the capability of a lens and of a photographic emulsion to reproduce sharply and accurately all the fine details in a picture.

\section{Films}

Photographs are produced by the action of light on a chemically prepared surface (film), which one must have some knowledge about in order to get the best results [14].

Types

- $\quad$ Black \& White

- Colour

- $\quad$ Colour reversal (for slides)

- Instant film (Polaroid)

The speed of the film indicates its sensitivity to light. According to the various systems, they are denoted by ISO, ASA, DIN, etcusually printed on the carton of each film. These films can easily be grouped as follows:

a) Ultra fast and fast films: having larger grains and less contrast.

b) Medium speed films: having fine grains and medium contrast.

c) Slow speed films: having very fine grains and medium to more contrast, though very slow.

For dental photography, the ideal film would be ISO 100 that provides adequate sharpness and detail. Light would not be a constraint in these situations as the conditions are static and well illuminated with a flash.

\section{Flash}

Modern flash units are a handy source of light for all occasions. These are of two types:

a) Flash gun with bulbs of different sizes, clear or blue.

b) Electric flash units, for several exposures without changing the bulb.

\section{Synchronization}

It's the automatic co-ordination between shutter speed and flash [13]. Flash must fire at the same time as the shutter opens, else, a picture on the film can't be obtained.

\section{' $\mathrm{X}$ ' and ' $\mathrm{M}$ ' contacts}

All cameras are provided with two contacts marked as ' $\mathrm{X}$ ' and ' $M$ ', ' $X$ ' synchronization is designed for the use of electronic flash at all shutter speeds while ' $M$ ' is for the use of flash bulbs only.

\section{Some useful accessories}

The camera kit bag may contain a number of accessories which increase the photographer's efficiency:

a) Change bag: Useful and handy, it's just like a portable darkroom in emergency. b) Bulk film loader: For safe handling and loading of $35 \mathrm{~mm}$ bulk film, this is another useful light-tight gadget made of bakelite or plastic.

c) Exposure meter: A small yet wonderful instrument for measuring the intensity of light falling on subject.

d) Cable release: A stiff wire in a flexible outer covering of metal or cloth with one end fitting into the camera shutter and the other end, a small knob which, when pressed, releases the shutter. It helps to avoid a 'camera shake'.

e) Stand or tripod: Firm and rigid stand to avoid 'camera shake'.

f) Lens hood: A sun-shade that shields extra light, other than that coming from the subject itself.

g) Supplementary lenses: Extra lenses of various focal lengths, which change or shorten the focal length of the camera lens just like a filter.

h) Red-eye: Red color comes from light that reflects off of the retina in eyes due to blood vessels nourishing the eye [14]. Many cameras have "red eye reduction" feature where flash goes off twice-once right before the picture is taken to contract people's pupils reducing red eye significantly and then again, to actually take the picture.

i) Light source: Broadly speaking, daylight and artificial light are two light sources for photography and must never be combined together to obtain natural colors in pictures. Strange unnatural color effects may sometimes be created intentionally.

j) Intra oral Cameras: An intra oral video system consists of camera itself, associated electronics, lighting system and monitor on which high-resolution image can be displayed [15]. They are of two types

1) Mouth mirror cameras: Fixed to video cameras and these are least problematic as hygiene is concerned. Endoscopic cameras with removable cap, which cover lens and possibly chip can be easily sterilized.

2) Endoscopic cameras: Hand pieces with small lens on tips.

\section{Digital Photography}

The buzzword today is digital. Be it music, T.V., video, watch, diary or any appliance, the world is going digital. The only difference is that the film is replaced with a CCD image sensor, thus, creating image digitally in camera without first chemically developing a latent image.

The finer the grid of the CCD, the greater is the amount of detail recorded. The basic unit of image detail is known as 'pixel' (picture element) [16-18].

Film less photography, pictures on a chip, call it what, this is a new phenomenon of technology. It is big and it is the way things will be from now on. 


\section{Advantages}

1) Makes the office paperless to a great extent (inexpensive storage digitally). Also, there is no ageing of photos.

2) Pictures can be viewed and the results can be confirmed immediately.

3) Images can be improved, color balanced, or for graphic artist the images can be manipulated with more freedom of artistic expression with no limitation to stretch the creative ability it can also get abused in the wrong hands (dental forgery) [19].

4) No wet processing is involved.

5) Easy retrieval and print outs whenever required.

6) Most cameras have video capture and output which are very helpful for multimedia presentations (Figure 6).

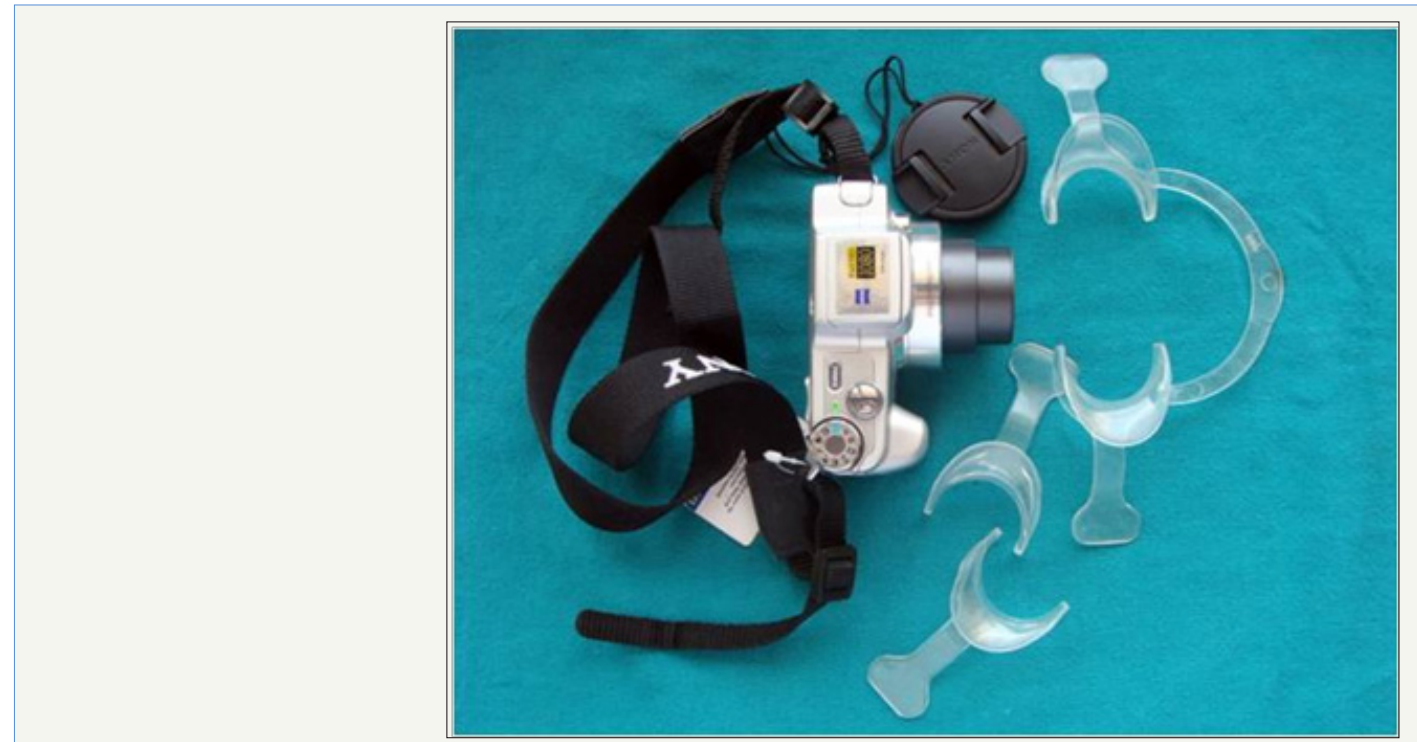

Figure 6: Digital camera with retractors.

7) Transmission across the world for communicating and video conferencing is feasible.

8) Understanding the basics: A series of lenses focus light onto a semiconductor device instead of onto a piece of film to create an image.

9) A computer takes this information down into digital data in its own language-bits and bytes. Essentially, a digital image is just a long string of $1 \mathrm{~s}$ and $0 \mathrm{~s}$ that represent all the tiny colored dots or pixels that collectively make up the image.

10) CCD has millions of pixels arranged in a grid pattern instantaneously.

11) The CCD is a semiconductor storage device where an electrical charge is moved across by signals. The presence or absence of charge denotes one or zero.

12) CCDs are monochromous, hence, can detect only black or white; one-shot colour is generally achieved with what is called a striped array. This involves filtering the individual pixels on the area array, alternating with red, green and blue.

13) In fact, the CCD chip in the digital camera is a triple-decker sandwich, a bottom slab consisting of silicon, with the CCD grid spread across it. The cells in the CCD layer correspond to the pixels of light, which determines how sharply the picture is recorded on top is a filter bank, which divides the light coming from the scene into the three primary colors red green and blue, and diverts it into the corresponding CCD cells.

14) There are 256 gray shades, and varying a level of each of the three colors results in the gamut of 16.7 million colors.

\section{5) IMAGE STORAGE (MEMORY CARDS)}

16) Almost all newer models have removable storage media, though the older models have built in storage. It's easy to replace card when full and insert another. Memory card capacity can vary from $2 \mathrm{MB}$ to $64 \mathrm{MB}$

17) Smart media, compact flash cards, memory sticks pc cards or even regular floppies are some examples to transfer images using adapter card reader for manipulation and storage of the image there.

18) The card reader can be plugged into serial port, parallel ports or USB ports.

19) There are provisions to compress the image (usually in the JPEG format) as each image will consume a considerable amount of camera's memory.

It must be noted that digital photography need not begin from digital cameras only but can also be produced from regular prints or films by digitizing them using scanners. Digital cameras have come a long way and are highly distinguishable from their traditional counterparts (Figure 7). 


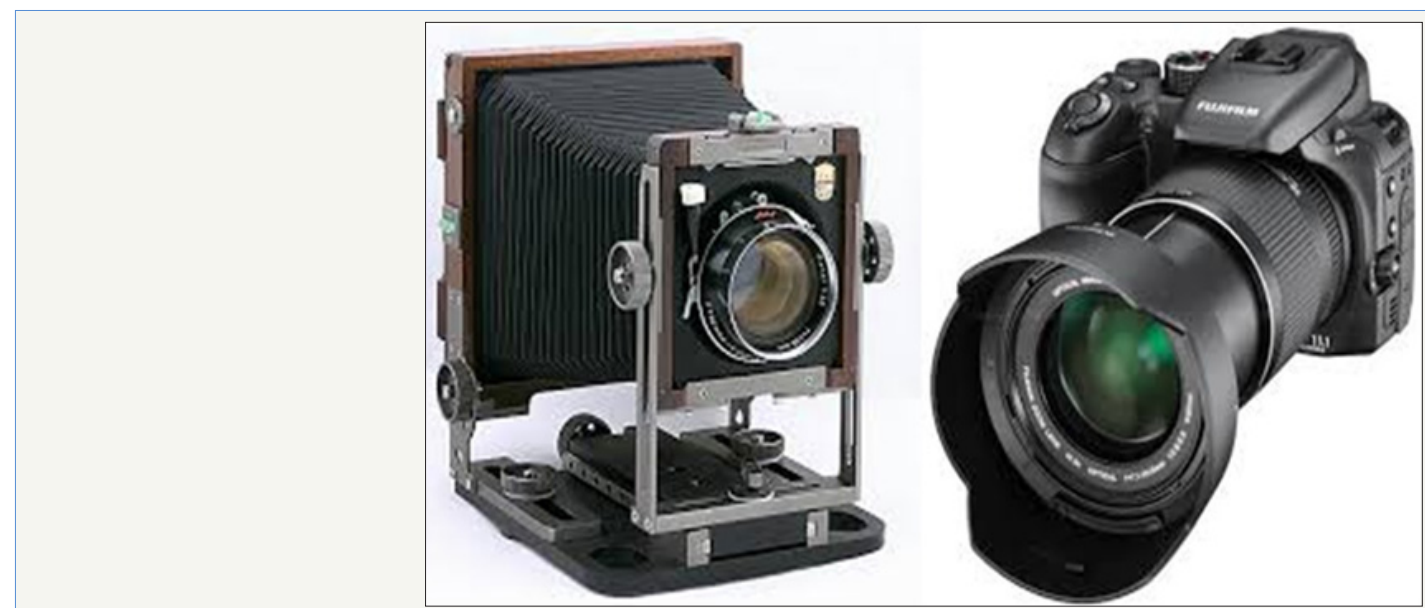

Figure 7: Traditional camera (left) Vs modern camera (right).

\section{Types of digital cameras}

1) Consumer point and shoot cameras:

a) Fully automatic

b) Have low resolution

c) $4 \times 6$ inches

2) Prosumer cameras

3) Based on the $35 \mathrm{~mm}$ model, they have range finder like viewing system and not SLR model

\section{4) Professional cameras}

These multi pixel cameras are SLR and hence accessories like macro lenses, ring flash etc can be used.

\section{Batteries and ac connection}

Some digital cameras use ordinary alkaline batteries and have a battery life of only 10-15 photographs. Others have rechargeable batteries that can last through more pictures. Digital cameras with two rechargeable batteries never allow the "no battery" situation. An external AC connection can be helpful even if it is not used routinely. The power cord tends to interfere with operator movement, and a socket must be available nearby.

\section{File format and software compression}

Once an image has been acquired by the CCD, it is stored in the camera's memory as a file. Image files can be of different formats and more important, can be compressed. Compression increases the number of images that can be stored in memory, but it also causes a decay of the image quality. The file storage format is not critical, but it is preferable to use digital cameras that save the acquired images as JPEG or TIFF files, which can be read by virtually any imaging software.

\section{Image storage}

Digital cameras with only internal memory should be avoided. Four types of removable memory are currently available for digital cameras: a) Solid state floppy disk card (SSFDC) or "smart card".

b) Miniature card

c) Compact flash card

d) 3.5" floppy disk

\section{Conclusion}

Photography is a universal means of communication and a valuable tool in many fields to record the things we see, as well as many subjects beyond our range of vision. Obtaining high quality photographs is often tough even in the hands of the most enthusiastic photographers. This article concludes with the aim of making the orthodontists more aware of the sophisticated equipment called a camera and its accessories without which, our orthodontic records are unfeasible.

\section{References}

1. Gavrilov D (2006) Photography in dentistry. Serbian Dent J 53: 253-260.

2. Spencer DA (1973) The focal dictionary of photographic technologies. Focal Press, USA, p. 454.

3. Eder JM (1945) History of photography, ( $4^{\text {th }}$ edn), Dover Publications, New York, USA, pp. 258-259.

4. (2016) History of photography. Encyclopedia Britannica Online, Encyclopedia Britannica.

5. Daniel M, Daguerre (2000) Heilbrunn timeline of art history. The Metropolitan Museum of Art, New York, USA.

6. Lester, Henry M (1935) Stereoscopic photography. The Leica Manual 1935: 249-255.

7. Tolmachev IA (2010) History of photography Part 1: The Beginning.

8. Maheshwari A, Williams DR (2001) Learning optics using vision. Biomedical Engineering Center for Visual Science University of Rochester, New York, USA, 5: 2014.

9. Ray SF (2002) Applied photographic optics: Lenses and optical systems for photography, film, video, electronic and digital imaging. Focal Press, USA.

10. Graber TM Patient photography in orthodontics. Angle Orthod 16(1,2): $17-43$. 
11. Kumar M, Modi TG, Patel J, Sathvara N (2014) Mastering camera systems in dentistry. International Journal of Dental Clinics 6(3).

12. Hamley, Samuel OP, Curran, Morven (1940) Color Photography. Dental Items 62: 1154

13. Sandler PJ (2000) Profiting from photography. Angle Society Newsletter 2000: 26-27.

14. Sandler PJ, Murray AM Recent developments in clinical photography. Br J Orthod 26(4): 269-274.

15. Gholston LR (1984) Reliability of an intra oral camera: utility for clinical dentistry and research. Am J Orthod 85(1): 89-93.
16. Desai V, Bumb D (2013) Digital dental photography: A Contemporary Revolution. Int J Clin Pediatr Dent 6(3): 193-196.

17. Mladenovic D, Mladenovic L (2010) Importance of digital dental photography in the practice of dentistry. Sci J Faculty Med in Nis 27(2): 75-79.

18. Terry DA, Snow SR, Mc Laren EA (2008) Contemporary dental photography: Selection and application. Compend Contin Educ Dent 29(8): 432-436.

19. Tsang A, Sweet D, Wood RE (1999) Potential for fraudulent use of digital radiography. J Am Dent Assoc 130(9): 1325-1329.

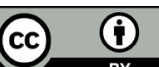

Creative Commons Attribution 4.0

International License

For possible submissions Click Here

\section{Submit Article}

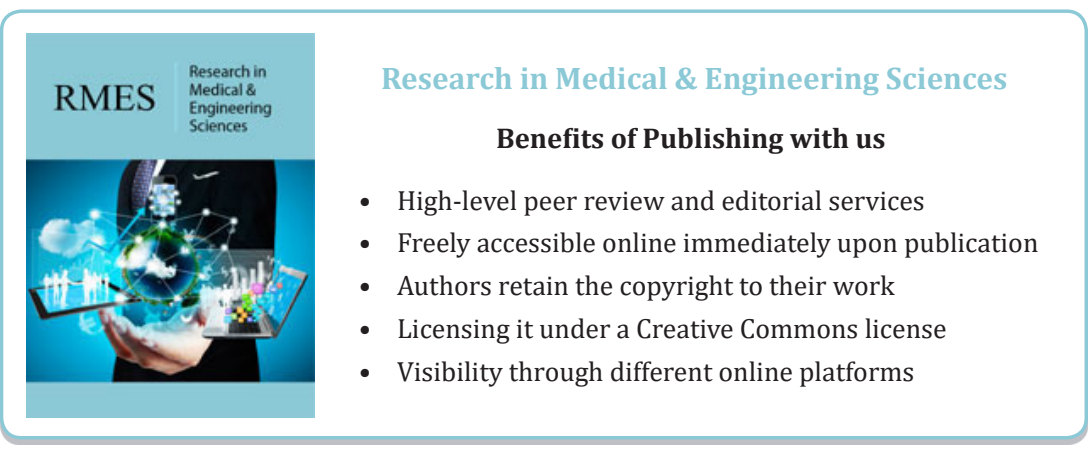

